\title{
Road Sign Recognition Based on Shape and Color Using SURF Method for Driver Assistance System
}

\author{
Muhammad Kamal Hossen ${ }^{1}$, Arifa Sultana ${ }^{1}$, Md. Shahnur Azad Chowdhury ${ }^{2}$ and \\ Md. Sajjatul Islam ${ }^{3}$ \\ ${ }^{1}$ Dept. of Computer Science and Engineering, CUET, Chittagong, Bangladesh \\ ${ }^{2}$ Dept. of Business Administration, IIUC, Chittagong, Bangladesh \\ ${ }^{3}$ Dept. of Computer Science and Engineering, CIU, Chittagong, Bangladesh \\ kamalcsecuet@gmail.com,arifa@baiust.edu.bd,tipu_iiuc@yahoo.com, \\ sajjatcse99@gmail.com
}

\begin{abstract}
The world is digitalizing day by day because of the contribution of science and technology. Intelligent Transport System (ITS) is a prominent innovation of this technology. It is an outstanding system which contributes not only to the betterment of human lives and environment but also to save time. Road sign recognition is one of the prominent sectors in the ITS. It provides real-time data on traffic for processing to take proper actions. This paper represents a color and shape based road sign detection model. As most of the road signs in Bangladesh are red; so, red channel of the image is extracted for detecting road sign accurately. A robust binarization technique is used to find the largest blob which indicates the region of interest (ROI). To extract ROI, the bounding box is used which is imposed on the RGB image. For decreasing computation time, the grayscale color space is used here. Then the features of the image are extracted and recognized by matching with the images in the database using Speed Up Robust Features (SURF) method. This method provides more accurate results in recognizing road signs. Various road sign images are used with a variety of conditions to test the proposed framework and results are presented to prove its effectiveness.
\end{abstract}

Keywords: RGB channel, Blob, Region of Interest (ROI), Bounding box, Speeded Up Robust Feature (SURF)

\section{Introduction}

People are highly dependent on the traffic systems from the eve of the creation of mankind. The car is playing a role model since the $20^{\text {th }}$ century. As technology is progressing day by day, people are now motivating to drive the automotive vehicles. The ITS technologies are being widely implemented to maximize the transportation safety and efficiency. It aims to manage factors such as cars, vehicles, loads, and routes to improve the safety and reduce the vehicle accidents and the fuel cost. The road sign recognition is an important part of ITS which offers ways to collect the real-time traffic data for processing with a central facility.

\section{Related Works}

There are so many works done on road sign detection applying the different methods. In the detection phase of $[1,5]$, the Hough transform algorithm is used to identify the shape of the road sign. The radial symmetry detection method is used in [2] which scan

Received (March 26, 2018), Review Result (May 1, 2018), Accepted (May 9, 2018)

* Corresponding Author 
for the shapes of one or more radii. The color segmentation based on histogram intersection for the efficiency matching model is described in the proposed method of [4]. The Single Pixel Voting (SPV) and Pair-Wise Pixels Voting (PWPV) schemes are used in [6] to detect road signs. The speeded up robust feature (SURF) descriptor which is invariant to rotation, skew, and occlusion of the sign, and the artificial neural network (ANN) classifier are used in [10] to detect and recognize the road signs. The purpose of this research is to develop a road sign recognition system for the automotive vehicles which detects the red colored road sign by extracting the red channel of the RGB image and recognize it using the SURF descriptor.

The organization of the rest of the paper is given as follows. Section 3 illustrates the proposed system using a flowchart. A sample dataset is given in Section 4. After this, the recognition along with the performance analysis of the classifier is described in Section 5. Finally, the conclusion and future works are mentioned in Section 6.

\section{The System Overview}

The proposed method for the road sign detection and recognition has several steps. The output of one step is the input of the next step. These steps are shown in the flowchart of Figure 1 and described in the following sections.

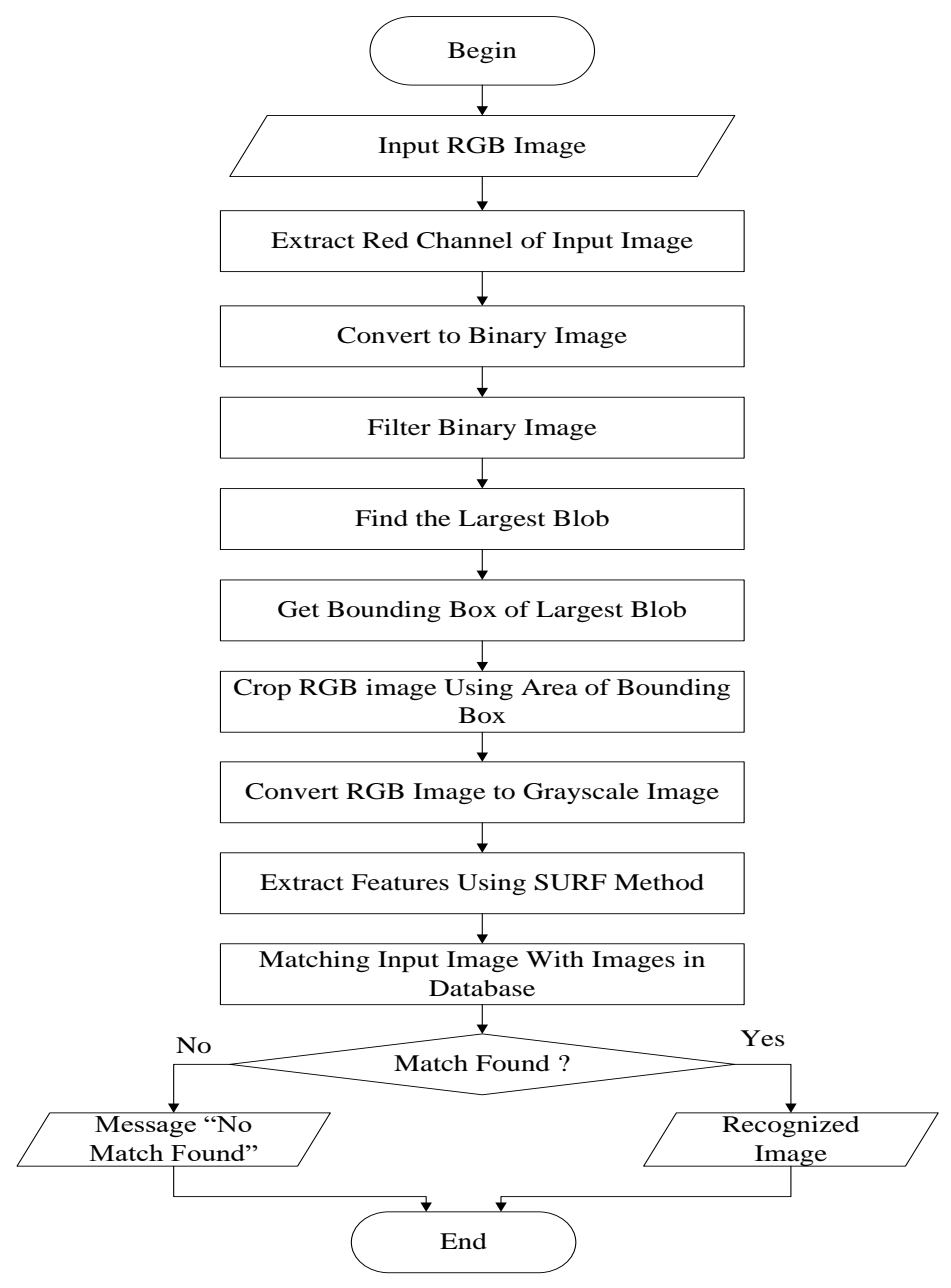

Figure 1. Flow Chart of the Proposed Method

\subsection{Image Acquisition}

In this phase, the RGB images with the road signs are taken using a digital camera. 


\subsection{Red Channel Extraction}

As the RGB images follow the additive color model, it consists of the red, green and blue color channels, each of which can be extracted according to the necessity. As stated earlier that most of the road signs in Bangladesh constitute with the red color. So, in the proposed method, the red channel of the input image is extracted for the optimization of the detection and recognition process of the road signs.

\subsection{Binarization}

The binarization of the input image is done by setting the red channel's value as a threshold. As a result, only those pixels in the input image which possess the intensity value of the red channel greater than the threshold value are converted to the foreground of the binary image. The other pixels are considered as the background.

\subsection{Filtering}

Filtering is a technique for modifying or enhancing an image. The image processing operations implemented with filtering include smoothing, sharpening, edge enhancement, and removal of noises. Filtering is a neighborhood operation in which the value of any given pixel in the output image is determined by applying some algorithm to the values of the pixels in the neighborhood of the corresponding input pixel. A pixel's neighborhood is some set of pixels defined by their locations relative to that pixel.

\subsection{Largest Blob Detection}

Each connected foreground region is known as the blob. Here, the area of each blob is calculated and these areas are sorted in the descending order. So, the first element of the array is the largest blob which is considered as the region of interest (ROI) as it has the high possibility to indicate the region of the road sign in the image.

\subsection{Finding Bounding Box of the Largest Blob}

By calculating the area of the largest blob i.e., the region of interest, the area of the bounding box is found which is the smallest area enclosing the region of interest. The area of a rectangular bounding box is calculated using the following equation:

area $=\left(s w_{-}\right.$longitude $-n e_{-}$longitude $) *\left(s w_{-}\right.$latitude - ne_latitude $)$

where $s w=$ South-West and $n e=$ North-West .

\subsection{Crop RGB Image Using the Bounding Box}

The bounding box which contains the area of the region of interest in the binary image is superimposed on the RGB input image. Then the area of the RGB image in the bounding box is cropped to extract the part of the image which contains only the road sign removing the background or environment. After that, the image is reshaped as like the shape of the images in the database. Thus, the road sign in the input image is detected finally.

\subsection{RGB to Grayscale Conversion}

The extracted part of the RGB image by the bounding box is converted to the grayscale image for faster processing. There are three methods for converting an RGB image to the grayscale image. The lightness method averages the most prominent and the least prominent colors i.e., $(\max (R, G, B)+\min (R, G, B)) / 2$. 
The average method simply averages the $\mathrm{R}, \mathrm{G}$, and $\mathrm{B}$ values i.e., $(R+G+B) / 3$. A more sophisticated version of the average method is the luminosity method. It also averages the values, but it forms a weighted average to account for human perception. We are more sensitive to green than other colors, so green is weighted most heavily [11]. The formula for luminosity is

$0.21 R+0.72 G+0.07 B$

\subsection{Feature Extraction Using the SURF Method}

The Speeded Up Robust Features (SURF) is a rotation and scale-invariant detector and descriptor which is used to extract the key features. The main purpose of the SURF method is to find the corresponding points between two images of the same object. The procedure of the SURF algorithm is divided into the three steps such as:

i) Detection of the interest key points,

ii) Feature vector estimation which is called the descriptor, and

iii) Matching between images.

The interest key points detection is deployed to find the relevant points in an image or object in order to trace the valuable information that needs to be computed by a local descriptor. The SURF method uses a blob detector to find the key points. The HessianLaplace matrix detector is used for this purpose. The Hessian matrix as stated in equation (3) is a second derivative matrix which detects the minima and the maxima based on the intensity of a specific region in the image. The SURF detector works for the nonmaximal-suppression of the determinants of the Hessian matrix. The Hessian matrix detects the key points and removes the weak edges.

$H=\left[\begin{array}{ll}I_{x x} & I_{x y} \\ I_{x y} & I_{y y}\end{array}\right]$

The Hessian matrix does not work for a single pixel. It is calculated for the vicinity (a patch of the image) around a pixel. Let $I(i)_{x x}, I(i)_{x y}$, and $I(i)_{y y}$ are the derivatives of a point $i_{0}$, then,

$H=\left[\begin{array}{cc}\sum i \in V I(i)_{x x} w\left(i-i_{0}\right) & \sum i \in V I(i)_{x y} w\left(i-i_{0}\right) \\ \sum i \in V I(i)_{x y} w\left(i-i_{0}\right) & \sum i \in V I(i)_{y y} w\left(i-i_{0}\right)\end{array}\right]$

Here, $w(t)$ is a Gaussian kernel. The equation (4) integrates the derivative values over the vicinity $V$ around the current pixel. Each value of the neighbors is multiplied by a value that shrinks as the distance increases. The law of decreasing follows Gaussian because $w(t)$ is Gaussian kernel centered at $i_{0}$.

The estimation of a feature vector finds out the potential regions within the neighborhood of the interest points. It has to be distinctive and at the same time robust to noise, geometric and photometric displacement deformations. Finally, the key points of the image are identified with labels, which can be detected using the locality sensitive hashing (LSH). It is an indexing scheme which performs an approximate searching in the high dimensional areas by computing all the nearest neighbors and choosing the nearest point. Figure 2 depicts the key points of the grayscale image which are extracted using the SURF feature detector. 


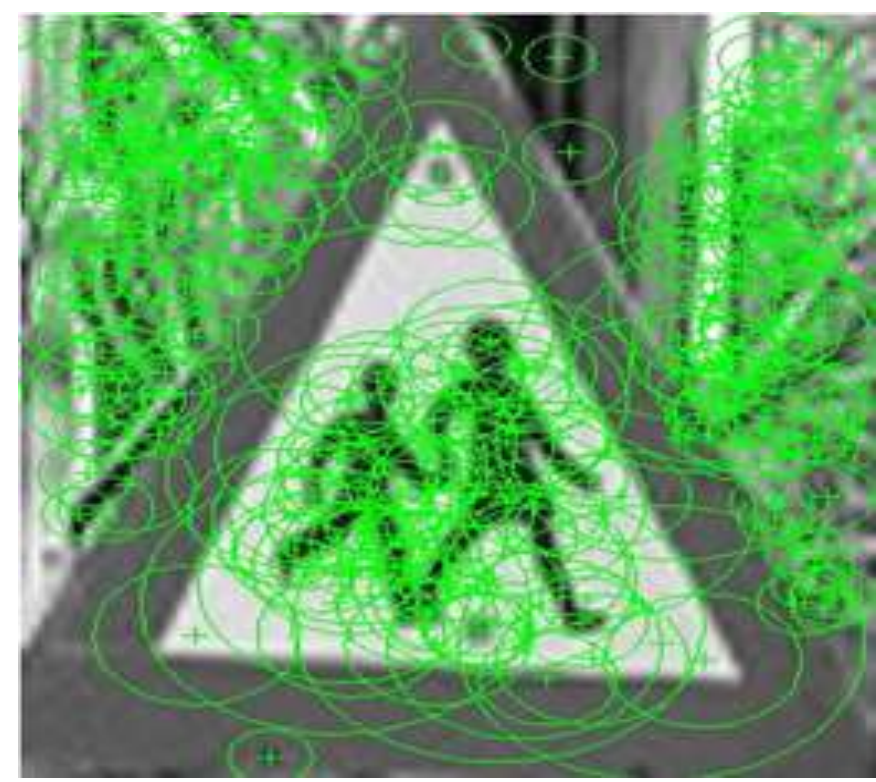

Figure 2. The Key Points Extraction of the Grayscale Image

\subsection{Matching Image with the Images of Database}

After finding the valid points of the input grayscale image, these points are compared with the valid points of the grayscale images in the database. To filter the matching results, the Euclidean distance between the matched descriptor and the most similar one is calculated at first. The smallest Euclidean distance between the analogous points constitutes a good match. The percentage of matching with each input image is calculated using the following equation:

$$
\text { Percentage of Match }=\frac{\text { Number of matched features points } * 100}{\text { Number of features points }}
$$

If the percentage of matching for all images is not equal to zero, the image in the database which has the maximum percentage value of the match is considered as the matched road sign. If the percentage of matching is zero for all the images in the database, then it is assumed that the input image is not a road sign. Thus the recognized road sign is shown in Figure 3.

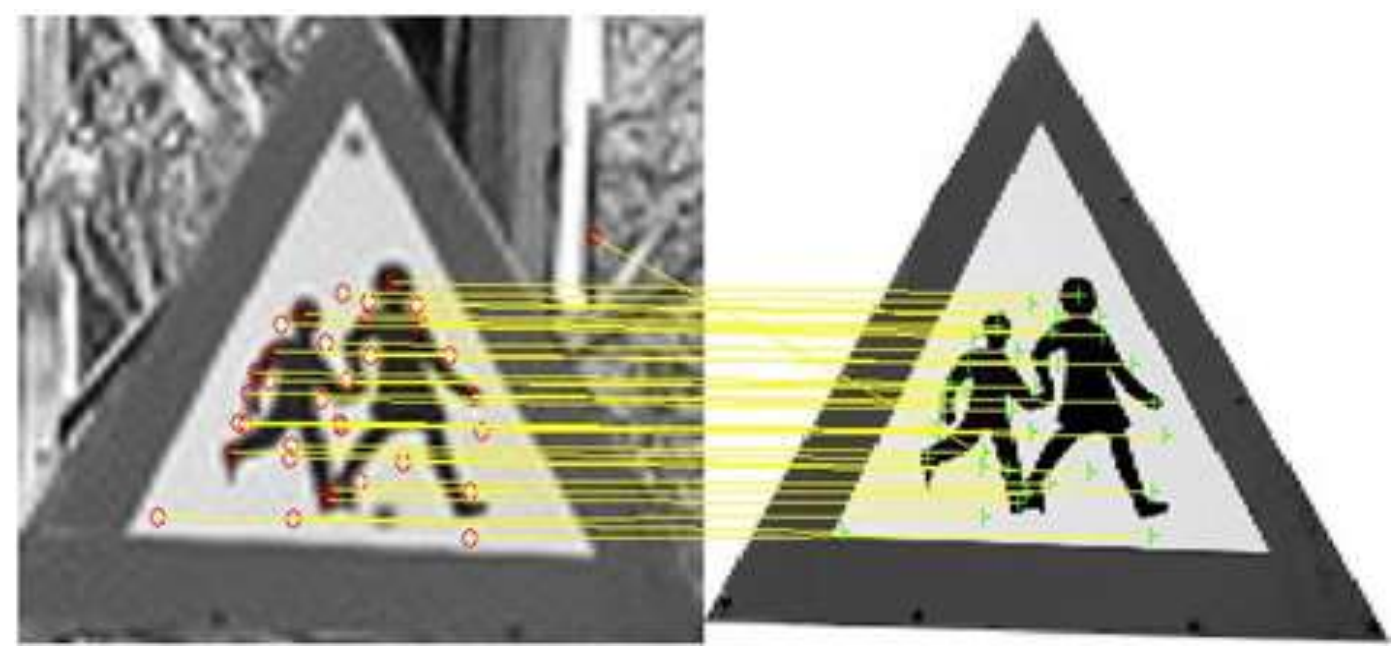

Figure 3. Matching Features Points with the Images in the Database 


\section{Dataset}

The input images are captured in different weather and illumination conditions. But it is difficult to create a large dataset in different environmental conditions. So, same road signs are captured at different times of a day i.e., at morning, noon, and late afternoon from the different distances and angles for different illumination conditions. Hence a dataset of 53 road sign images is formed. Some of them are shown in Figure 4.

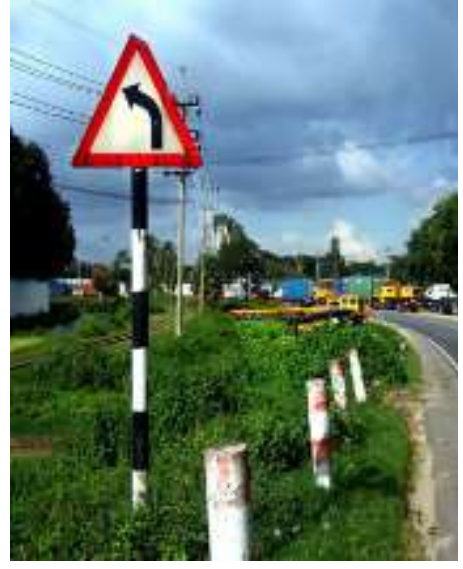

(a)

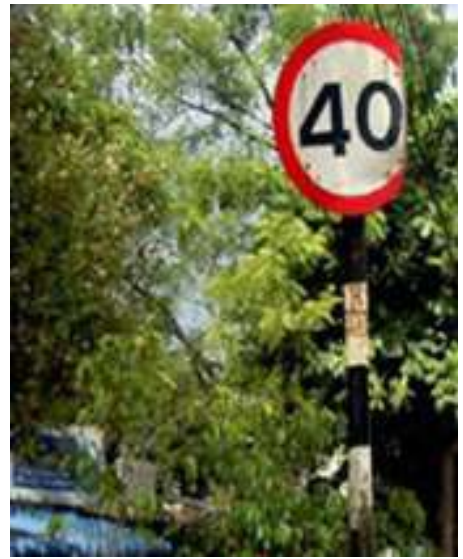

(d)

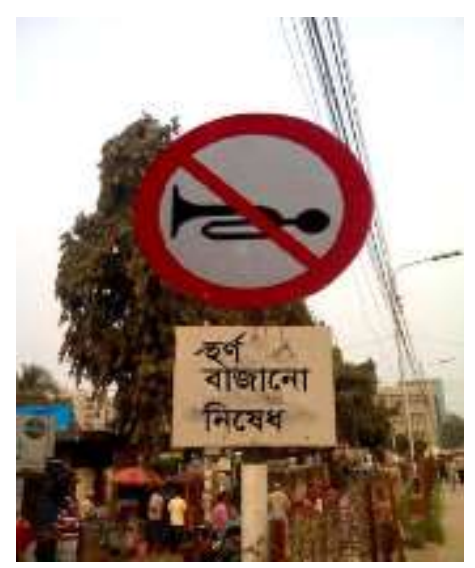

(g)

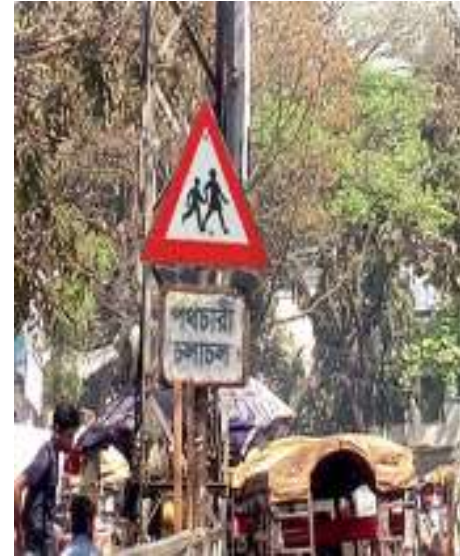

(b)

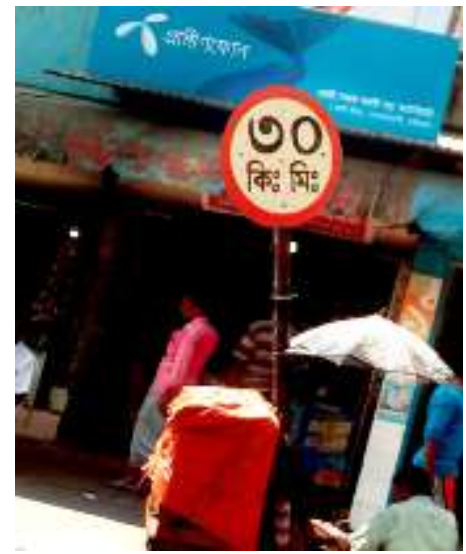

(e)

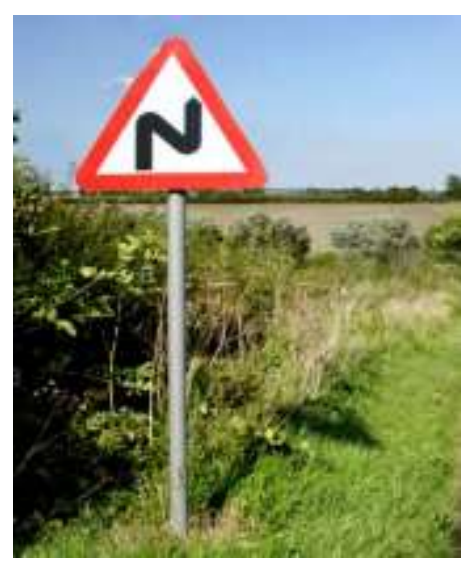

(h)

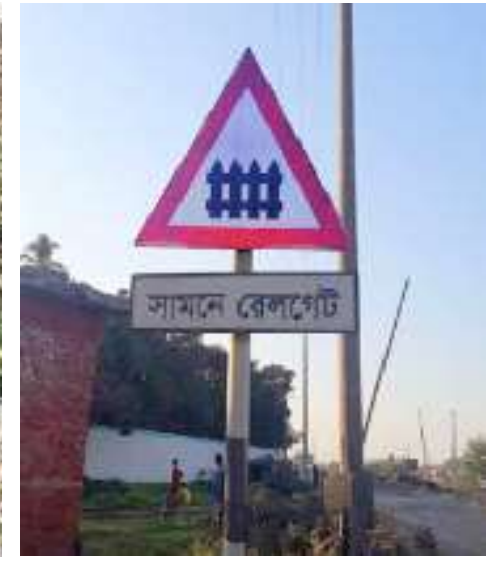

(c)

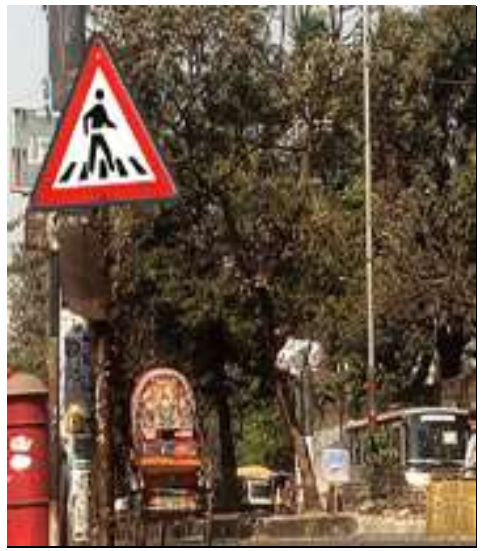

(f)

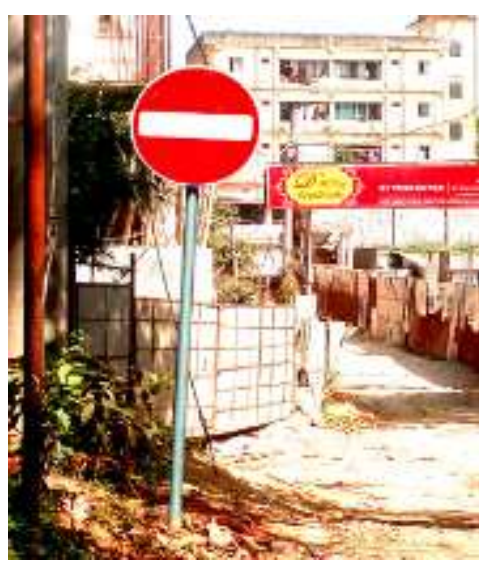

(i)

Figure 4. Sample Input Images from the Dataset 


\section{Experimental Results and Performance Analysis}

All experiments are done on the Core-i3 $2.53 \mathrm{GHz}$ processor with 2GB RAM under the MATLAB environment. The complete testing database consists of 53 digital road sign images. The experiments have been done using these road sign images. For each sample image, (a) the corresponding red channel image, (b) the binary image, (c) the grayscale image of the detected road sign, and (d) the recognized image of the road sign from the database are identified. Finally, the meaning of the recognized road sign is shown in the command window. An example of the processing along with the input image to detect the triangular road sign is shown in Figure 5. The meaning of the recognized road sign is "Roads for Pedestrian".

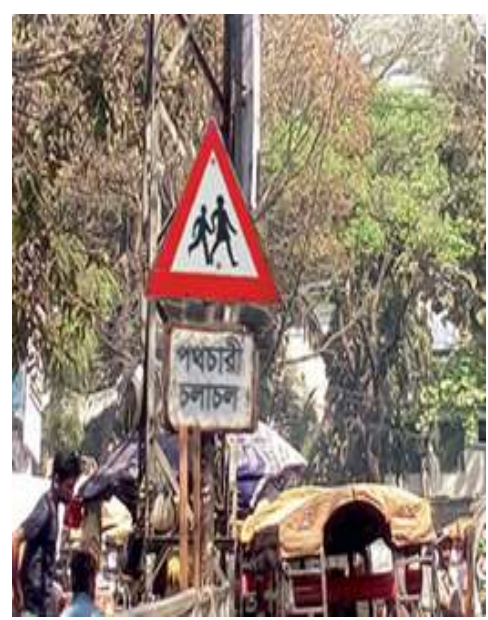

(a)

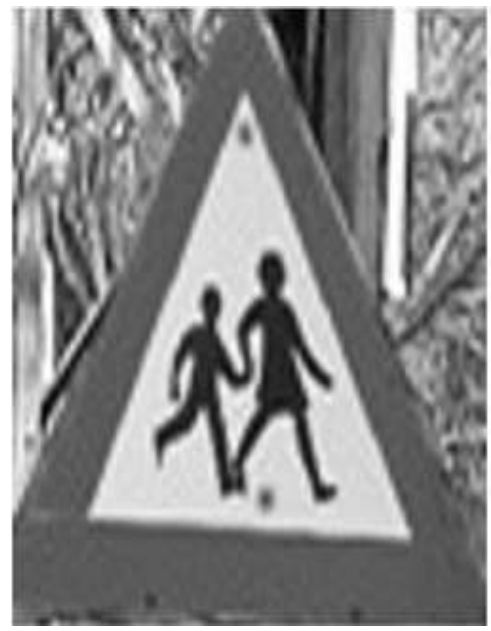

(d)

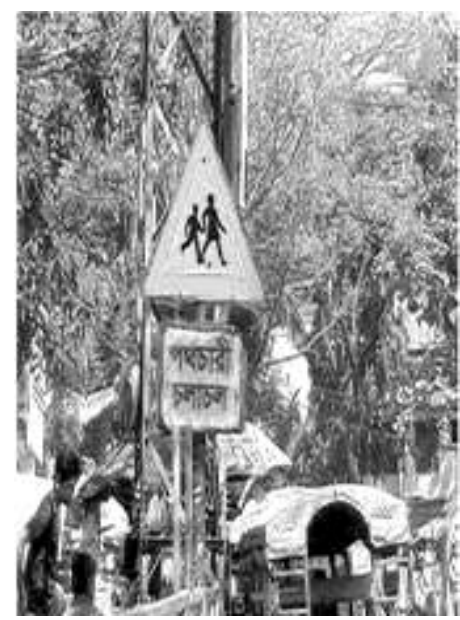

(b)

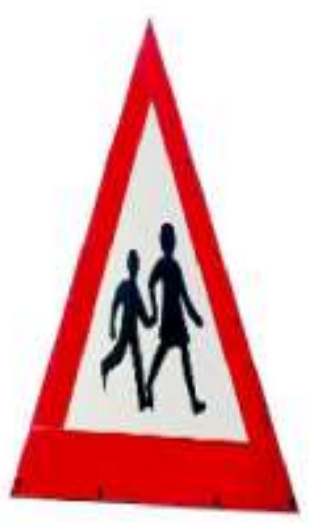

(e)

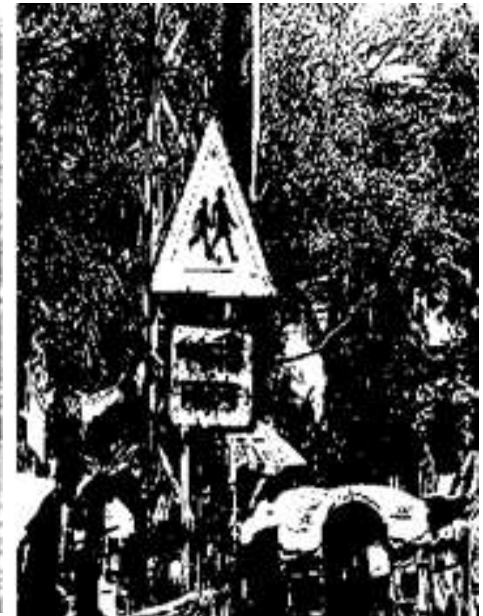

(c)

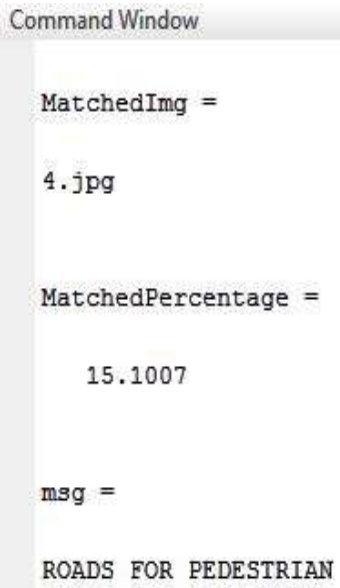

(f)

Figure 5. (a) Input RGB Image, (b) Input Image after Red Channel Extraction, (c) Binary Image, (d) Detected Road Sign, (e) Recognized Road Signs, and (f) Meaning of Road Signs in the Command Window

Another example of detection of the circular road sign with the processing steps is shown in Figure 6. The meaning of the recognized road sign is "Highest Speed Limit 40". 


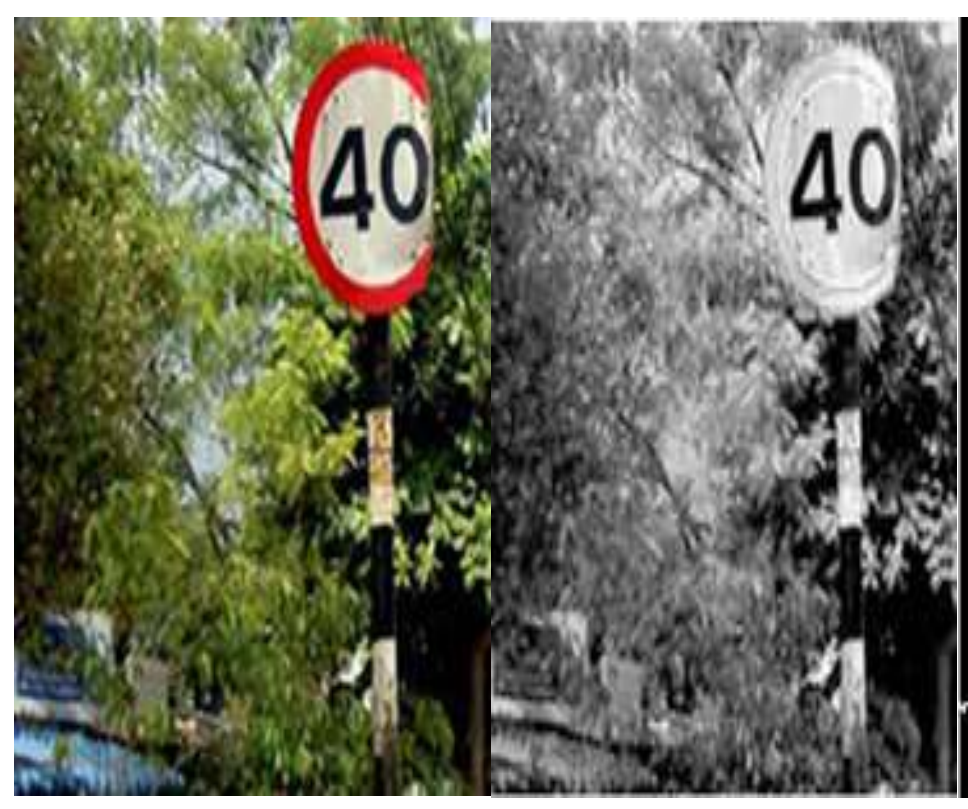

(a)

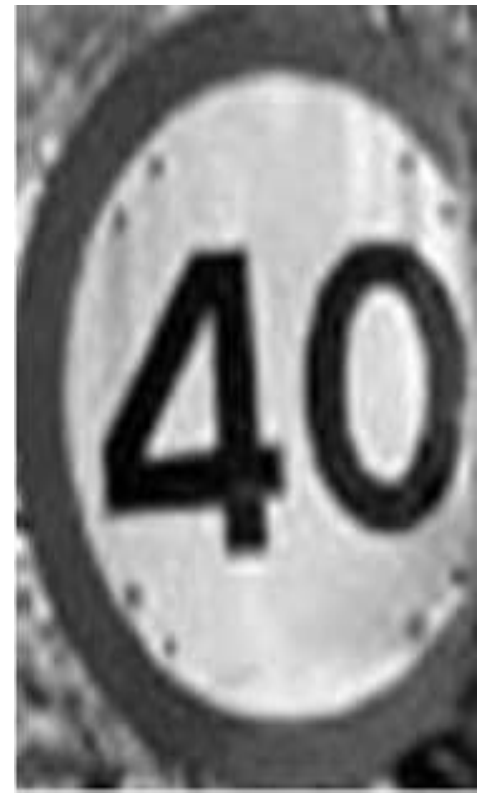

(d) (b)

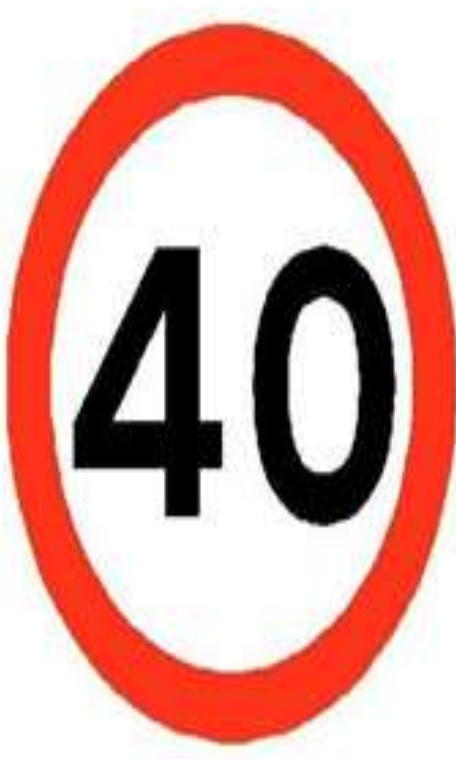

(e)

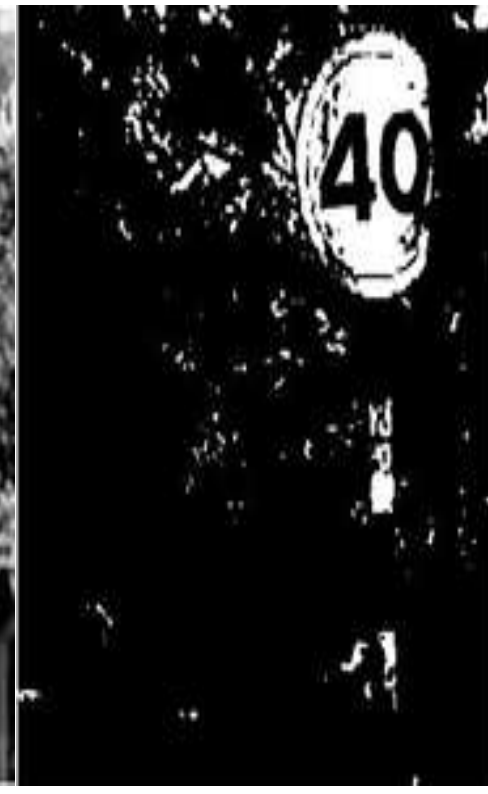

(c)

Command Window

MatchedIng =

13.Jpg

MatchedPercentage *

9.1837

misg =

HIGHEST SPEEO LIMIT 40

(f)

Figure 6. (a) Input RGB Image, (b) Input Image after Red Channel Extraction, (c) Binary Image, (d) Detected Road Sign (e) Recognized Road Signs, and (f) Meaning of Road Signs in the Command Window

There are some images of the road signs which cannot be detected accurately by this algorithm because of the different illumination conditions, large distances, blur in the images and too much complex background. Some of these are shown in Figure 7. 


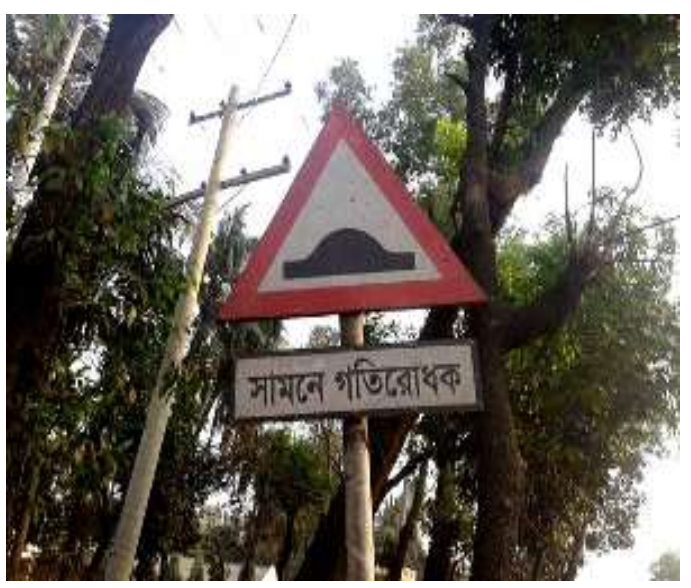

(a)

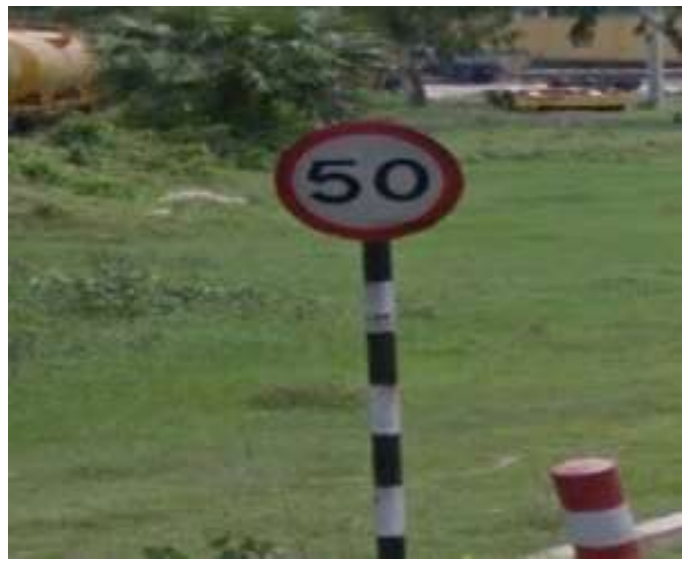

(c)

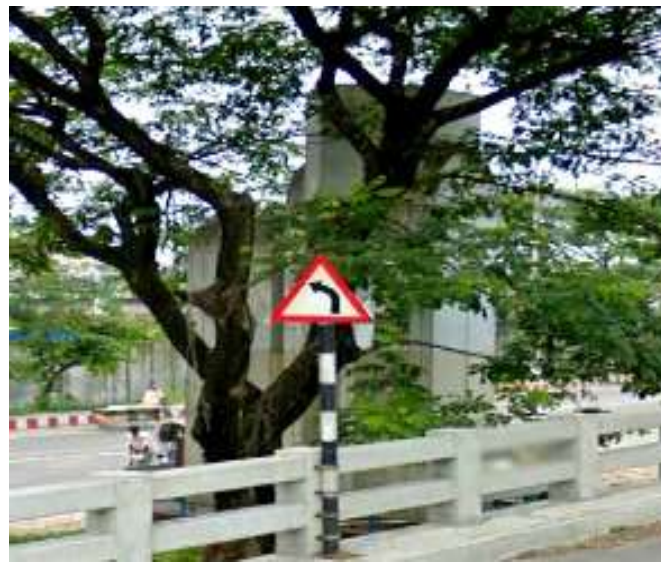

(b)

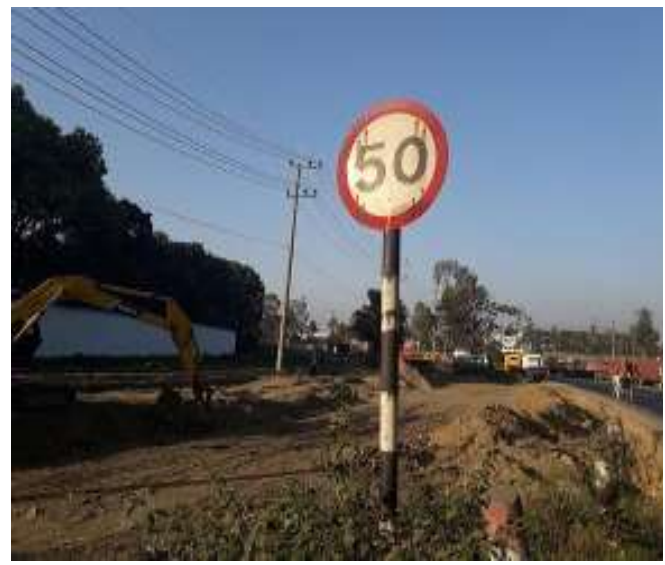

(d)

Figure 7. Sample Images Where Algorithm Failed to Recognize Road Sign

Comparing with the images in the database and calculating the matching percentage, the road signs in the input images are recognized. Here, there are total 53 input road sign images and the road signs are correctly recognized in 47 images. The accuracy of recognizing road signs in the simple and complex background is shown in Table 1.

Table 1. Experimental Result of Road Sign Detection and Recognition

\begin{tabular}{|c|c|c|c|}
\hline Background & No. of Input Test Images & No. of Recognized Images & Accuracy (\%) \\
\hline Simple & 22 & 20 & 90.90 \\
\hline Complex & 31 & 27 & 87.09 \\
\hline Overall & $\mathbf{5 3}$ & $\mathbf{4 7}$ & $\mathbf{8 8 . 9 9}$ \\
\hline
\end{tabular}

When the distance between the camera and the road signs is increased, the accuracy of recognizing road signs is decreased. This is shown in tabular form in Table 2 and in graphical form in Figure 8.

Table 2. Distance vs Accuracy Rate of Recognition

\begin{tabular}{|c|c|c|c|}
\hline Distance (Meter) & No. of Input Images & No. of Recognized Images & Accuracy (\%) \\
\hline 2 & 12 & 12 & 100 \\
\hline 3 & 10 & 8 & 80.00 \\
\hline 4 & 17 & 16 & 94.11 \\
\hline 5 & 14 & 11 & 78.57 \\
\hline
\end{tabular}


It can be seen from the distance versus accuracy curve of Figure 8 that the accuracy rate becomes maximum when the distance is 2 meters. The accuracy rate is decreasing when the distance is being greater than the 4 meters. So, we can use zoom in/out parameter of the digital camera for keeping the distance nearest to 4 meters.

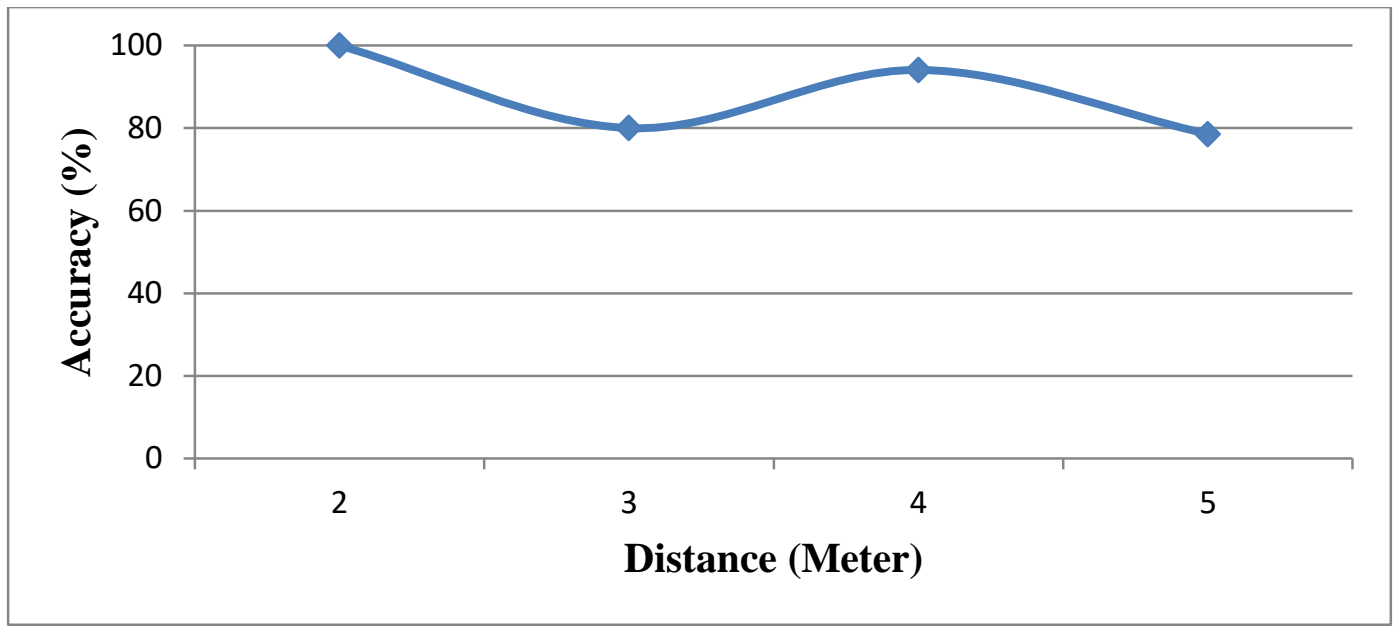

Figure 8. Distance vs Accuracy Rate of Recognition

\section{Conclusion and Future Work}

In this paper, an algorithm is introduced for detecting and recognizing the road sign from the input road sign images. This helps the autonomous systems to perform driving accurately and safely in the driverless vehicles or to assist a driver to drive consciously in the unknown environments. The proposed algorithm is successfully tested by a group of images with the various environments. These images are taken under various illumination conditions. It successfully detects the road signs in the input road sign images and recognizes them with acceptable accuracy.

The proposed algorithm has a limitation of detecting the blue colored road signs in the input images. Another limitation of this algorithm is that it cannot detect too much-tilted road sign. It can detect road sign under different illumination conditions. But when the environment is highly illuminated, it cannot recognize the road sign accurately.

In the future, this work can be extended to add some features and improve performances such as

Improve the algorithm for detecting and recognizing more red and blue colored road signs from the input images.

$>$ Make capable to recognize the tilted road signs from the input images which are captured from the vehicles in motion.

\section{References}

[1] S. S. M. Sallah, F. A. Hussin and M. Z. Yusoff, "Shape-based Road Sign Detection and Recognition for Embedded Application Using MATLAB", Proceedings of the International Conference on Intelligent and Advanced Systems (ICIAS), (2010) July, DOI: 10.1109/ICIAS.2010.5716193.

[2] G. K. Siogkas and E. S. Dermatas, "Detection, Tracking and Classification of Road Signs in Adverse Conditions", Proceedings of the IEEE Mediterranean Electrotechnical Conference (MELECON), Spain, (2006) May, pp. 537-540, DOI: 10.1109/MELCON.2006.1653157.

[3] Y. Aoyagi and T. Asakura, "A Study on Traffic Sign Recognition in Scene Image Using Genetic Algorithms and Neural Networks", Proceedings of the IEEE International Conference on Industrial Electronics, Control, and Instrumentation (IECON), Taiwan, vol. 3, (1996) August, pp. 1838-1843, DOI: 10.1109/IECON.1996.570749.

[4] M. J. Swain and D. H. Ballard, "Indexing via Color Histograms", Proceedings of the Third International Conference on Computer Vision, Japan, (1990) December, pp. 390-393. DOI: 10.1109/ICCV.1990.139558 
[5] A. Hechri and A. Mtibaa, "Lanes and Road Signs Recognition for Driver Assistance System", International Journal of Computer Science Issues (IJCSI), vol. 8, issue 6, no. 1, (2011) November, pp. 402-408.

[6] H. Fleyeh and M. Dougherty, "Road and Traffic Sign Detection and Recognition", Proceedings of the $10^{\text {th }}$ EWGT Meeting and $16^{\text {th }}$ Mini-EURO Conference, (2005), pp. 644-653.

[7] M. L-. Calleja, T. Nagai, M. Attamimi, M. N-. Miyatake and H. P-. Meana, "Object Detection Using SURF and Superpixels", Journal of Software Engineering and Applications, vol. 6, (2013) September, pp. 511-518.

[8] U. Singh, S. S. Singh and M. K. Srivastava, "Object Detection and Localization Using SURF Supported by KNN", International Journal of Computer Science Trends and Technology (IJCST), vol. 3, no. 2, (2015) April, pp. 88-93.

[9] M. S. Hossain and Z. Hyder, "Traffic Road Sign Detection and Recognition for Automotive Vehicles", International Journal of Computer Applications (IJCA), vol. 120, no. 24, (2015) June, pp. 10-15.

[10] M. Z. Abedin, P. Dhar and K. Deb, "Traffic Sign Recognition Using SURF: Speeded Up Robust Feature Descriptor and Artificial Neural Network Classifier", Proceedings of the $9^{\text {th }}$ International Conference on Electrical and Computer Engineering (ICECE), Bangladesh, (2016) December, pp. 198-201, DOI: 10.1109/ICECE.2016.7853890.

[11] J. D. Cook, "Three Algorithms for Converting Color to Grayscale", [Online]. Available: https://www.johndcook.com/blog/2009/08/24/algorithms-convert-color-grayscale/.

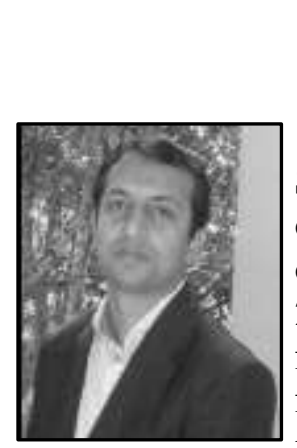

\section{Authors}

Muhammad Kamal Hossen, he has received his B. Sc. and M. Sc. in Computer Science \& Engineering degrees from the department of Computer Science \& Engineering (CSE) of Chittagong University of Engineering \& Technology (CUET), Bangladesh in 2005 and 2015 , respectively. He is now pursuing his $\mathrm{Ph}$. D. degree in CSE from the same university. Since 2006 , he has been serving as a faculty member in the Department of CSE, CUET. His research interests include digital image processing, cryptography, steganography, pattern recognition, and data mining.

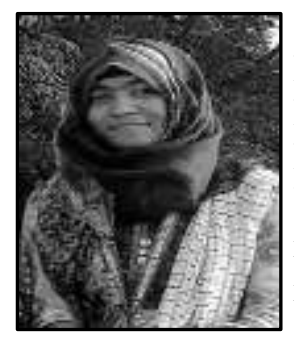

Arifa Sultana, she received her B. Sc. in Computer Science \& Engineering degree from Department of Computer Science and Engineering of CUET), Bangladesh in 2017. She is now serving as a Lecturer in the Department of CSE of Bangladesh Army International University of Science and Technology (BAIUST), Bangladesh. His research interests include digital image processing, pattern recognition, artificial intelligence.

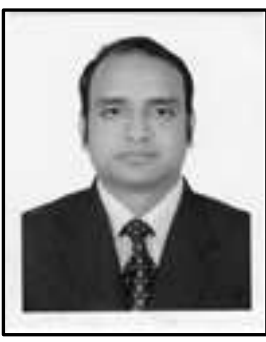

Md. Shahnur Azad Chowdhury, he received B. Sc. in Computer Science and Engineering from International Islamic University Chittagong (IIUC), Bangladesh in 2003 and M. Sc. in 2012 from Daffodil Int'l University, Dhaka, Bangladesh. He has been serving the IIUC for the last fourteen years as a Lecturer, Assistant Professor and Associate Professor, respectively. His areas of research interest are natural language processing, digital image processing, data mining, IoT. 


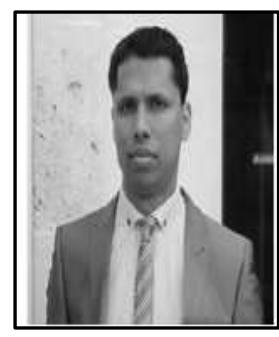

Md. Sajjatul Islam, he has received his B. Sc. in Computer Science \& Engineering (CSE) degree from the department of CSE of Chittagong University of Engineering \& Technology (CUET), Bangladesh in 2005 and Master of Science in Computing from University of Wales (UoW), United Kingdom in 2011. He is pursuing his $\mathrm{Ph}$. D. degree in CSE from CUET. He is now serving as an Assistant Professor in the Department of CSE of Chittagong Independent University (CIU), Bangladesh. His research interests include big data analysis, pattern recognition, cloud computing. 